\title{
Light-Front Quantization with Explicit Lorentz Symmetry for Yukawa Model
}

Received: 30 September 2014 / Accepted: 11 November 2014 / Published online: 21 December 2014 (C) The Author(s) 2014. This article is published with open access at Springerlink.com

\begin{abstract}
The Dirac method for constrained systems is incomplete for the light-front (LF) quantization of the Yukawa model in $D=1+1$ dimensions. A novel quantization procedure is proposed, where one obtains the LF commutator and anti-commutators directly from the Heisenberg equations generated by $P^{+}$, which is a kinematical operator. By adding the general assumptions on the quantum field theory, one evalutes 2-point Wightman functions for a free field case. The Lorentz symmetry is manifest at every step of this novel LF procedure. The Gaussian effective potential is defined with the point-splitting regularization with a space-like separation. The optimum values of the mass parameters are regularization independent.
\end{abstract}

\section{Novel LF Quantization}

The standard LF quantization [1-3] is the canonical quantization procedure for constrained systems. However, as it is shown in "Appendix B", the Dirac procedure [4-6] is incomplete at the LF hypersurface for fermion fields. Therefore we propose a novel procedure, which incorporates special advantages of the LF formulation. We may omit the classical canonical structure and start at the quantum level with the Heisenberg equations with $P^{+}$operator. This will allow us to read out the commutation relations for these fields, which are canonical at the LF hypersurface. Then by implementing some general properties of the quantum field theory we may evaluate the Wightman functions for the canonical fields and then also for those fields, which are not canonical.

We illustrate this procedure by considering the Yukawa model in $D=1+1$ dimensions, where the Lorentz invariant Lagrangian density is

$$
\begin{aligned}
\mathscr{L} & =\partial_{+} \phi \partial_{-} \phi-\frac{m^{2}}{2} \phi^{2}+i \bar{\Psi} \gamma^{\mu} \partial_{\mu} \Psi-(M+g \phi) \bar{\Psi} \Psi \\
& =\partial_{+} \phi \partial_{-} \phi-\frac{m^{2}}{2} \phi^{2}+i \sqrt{2} \psi_{+}^{\dagger} \partial_{+} \psi_{+}+i \sqrt{2} \psi_{-}^{\dagger} \partial_{-} \psi_{-}-(M+g \phi)\left(\psi_{+}^{\dagger} \psi_{-}+\psi_{-}^{\dagger} \psi_{+}\right) .
\end{aligned}
$$

From this Lagrangian we find two components of the canonical energy-momentum tensor

$$
T^{++}=i \sqrt{2} \psi_{+}^{\dagger} \partial_{-} \psi_{+}+\left(\partial_{-} \phi\right)^{2}, \quad T^{+-}=-i \sqrt{2} \psi_{-}^{\dagger} \partial_{-} \psi_{-}+(M+g \phi)\left(\psi_{+}^{\dagger} \psi_{-}+\psi_{-}^{\dagger} \psi_{+}\right)+\frac{m^{2}}{2} \phi^{2}
$$

J. A. Przeszowski $(\varangle) \cdot$ J. Żochowski

Faculty of Physics, University of Białystok, ul. Lipowa 41, 15-424 Białystok, Poland

E-mail: j.przeszowski@uwb.edu.pl

J. Żochowski

E-mail: jazo@alpha.uwb.edu.pl 
The $P^{+}=P_{-}$is a kinematical operator, which we define as the integral $P_{-}=\int_{\mathbb{R}} d x^{-} T^{++}$, thus the Heisenberg equations at fixed $x^{+}$LF time are

$$
\begin{aligned}
i \partial_{-} \psi_{+}^{\dagger}(x) & =\left[\psi_{+}^{\dagger}(x), P_{-}\right]=i \sqrt{2} \int_{\mathbb{R}} d y^{-}\left[\psi_{+}^{\dagger}\left(x^{+}, x^{-}\right), \psi_{+}^{\dagger}\left(x^{+}, y^{-}\right) \partial_{-} \psi_{+}\left(x^{+}, y^{-}\right)\right], \\
i \partial_{-} \psi_{+}(x) & =\left[\psi_{+}(x), P_{-}\right]=i \sqrt{2} \int_{\mathbb{R}} d y^{-}\left[\psi_{+}\left(x^{+}, x^{-}\right), \psi_{+}^{\dagger}\left(x^{+}, y^{-}\right) \partial_{-} \psi_{+}\left(x^{+}, y^{-}\right)\right], \\
i \partial_{-} \phi(x) & =\left[\phi(x), P_{-}\right]=2 \int_{\mathbb{R}} d y^{-}\left[\phi\left(x^{+}, x^{-}\right), \partial_{-} \phi\left(x^{+}, y^{-}\right)\right] \partial_{-} \phi\left(x^{+}, y^{-}\right) .
\end{aligned}
$$

From these relations we directly read out the non-vanishing LF canonical (anti)-commutators

$$
\left\{\psi_{+}\left(x^{+}, x^{-}\right), \psi_{+}^{\dagger}\left(x^{+}, y^{-}\right)\right\}=\frac{1}{\sqrt{2}} \delta\left(x^{-}-y^{-}\right), \quad 2\left[\phi\left(x^{+}, x^{-}\right), \partial_{-} \phi\left(x^{+}, y^{-}\right)\right]=i \delta\left(x^{-}-y^{-}\right) .
$$

Therefore in this model $\phi, \psi_{+}, \psi_{+}^{\dagger}$ are the LF canonical quantum fields. We assume that there exists one vacuum state, which is both the Lorentz and PCT invariant state and its energy-momentum eigenvalues vanish

$$
U(\omega)|0\rangle=|0\rangle, \quad \theta^{\mathscr{P} \mathscr{C} \mathscr{T}}|0\rangle=|0\rangle, \quad P^{ \pm}|0\rangle=0 .
$$

Next the translational invariance leads to the relations for the 2-point Wightman functions

$$
\langle 0|\phi(x) \phi(y)| 0\rangle=\left\langle 0\left|\phi(0) e^{-i P \cdot(x-y)} \phi(0)\right| 0\right\rangle, \quad\langle 0|\Psi(x) \bar{\Psi}(y)| 0\rangle=\left\langle 0\left|\Psi(0) e^{-i P \cdot(x-y)} \bar{\Psi}(0)\right| 0\right\rangle .
$$

Since $P^{ \pm}$operators have non-negative spectra, then (6) leads to the analyticity of 2-point Wightman functions as $x^{ \pm}-y^{ \pm} \rightarrow x^{ \pm}-y^{ \pm}-i 0$. The Lorentz transformation for scalar and fermion fields leads to

$$
\begin{aligned}
\left\langle 0\left|\phi\left(x^{+}, x^{-}\right) \phi(0)\right| 0\right\rangle & =\left\langle 0\left|\phi\left(x^{+} e^{\omega}, x^{-} e^{-\omega}\right) \phi(0)\right| 0\right\rangle, \\
\left\langle 0\left|\Psi\left(x^{+}, x^{-}\right) \bar{\Psi}(0)\right| 0\right\rangle & =S^{-1}(\omega)\left\langle 0\left|\Psi\left(x^{+} e^{\omega}, x^{-} e^{-\omega}\right) \bar{\Psi}(0)\right| 0\right\rangle S(\omega) .
\end{aligned}
$$

These general properties allow us to evaluate the Wightman functions for the LF canonical fields. First for the scalar fields we find

$$
\left\langle 0\left|\phi(x) \partial_{-} \phi(y)\right| 0\right\rangle=-i\left\langle 0\left|\phi(x)\left[\phi(y), P_{-}\right]\right| 0\right\rangle=i\left\langle 0\left|\phi(0) e^{-i P \cdot(x-y)} P_{-} \phi(0)\right| 0\right\rangle=-\left\langle 0\left|\partial_{-} \phi(x) \phi(y)\right| 0\right\rangle .
$$

Then for the fermions fields we need to implement the PCT transformation

$$
\left\langle 0\left|\psi_{+}^{\dagger}(x) \psi_{+}(y)\right| 0\right\rangle \stackrel{\mathscr{P} \mathscr{C} \mathscr{T}}{=}\left\langle 0\left|\psi_{+}(-y) \psi_{+}^{\dagger}(-x)\right| 0\right\rangle=\left\langle 0\left|\psi_{+}(0) e^{i P \cdot(y-x)} \psi_{+}^{\dagger}(0)\right| 0\right\rangle=\left\langle 0\left|\psi_{+}(x) \psi_{+}^{\dagger}(y)\right| 0\right\rangle .
$$

Thus the LF canonical (anti)-commutators (4) lead to the relations between the Wightman functions

$$
\begin{aligned}
-\left\langle 0\left|\partial_{-} \phi\left(x^{+}, x^{-}\right) \phi\left(x^{+}, y^{-}\right)\right| 0\right\rangle-\left\langle 0\left|\partial_{-} \phi\left(x^{+}, y^{-}\right) \phi\left(x^{+}, x^{-}\right)\right| 0\right\rangle & =\frac{i}{2} \delta\left(x^{-}-y^{-}\right), \\
\left\langle 0\left|\psi_{+}\left(x^{+}, x^{-}\right) \psi_{+}^{\dagger}\left(x^{+}, y^{-}\right)\right| 0\right\rangle+\left\langle 0\left|\psi_{+}\left(x^{+}, y^{-}\right) \psi_{+}^{\dagger}\left(x^{+}, x^{-}\right)\right| 0\right\rangle & =\frac{1}{\sqrt{2}} \delta\left(x^{-}-y^{-}\right),
\end{aligned}
$$

while the analyticity leads directly to the Wightman functions for canonical fields at equal LF time $x^{+}$

$$
\partial_{-}^{x}\left\langle 0\left|\phi\left(x^{+}, x^{-}\right) \phi\left(x^{+}, 0\right)\right| 0\right\rangle=-\frac{1}{4 \pi} \frac{1}{x^{-}-i 0}, \quad\left\langle 0\left|\psi_{+}\left(x^{+}, x^{-}\right) \psi_{+}^{\dagger}\left(x^{+}, 0\right)\right| 0\right\rangle=\frac{1}{\sqrt{2}} \frac{1}{2 \pi i} \frac{1}{x^{-}-i 0},
$$

where the Heisenberg distribution is denoted as

$$
\frac{1}{x-i 0}=\mathscr{P} \frac{1}{x}+i \pi \delta(x) \text {. }
$$

For the later convenience we introduce notation for the Wightman functions for scalar and fermion fields

$$
\left\langle 0\left|\phi\left(x^{+}, x^{-}\right) \phi(0)\right| 0\right\rangle=S_{\phi \phi}\left(x^{+}, x^{-}\right), \quad\left\langle 0\left|\psi_{a}(x) \psi_{b}^{\dagger}(y)\right| 0\right\rangle=S_{a b}(x-y), \quad a, b \in\{+,-\}
$$




\section{Volterra Equations for Wightman Functions}

In the next step of our analysis we use the equations of motions for fields, which follow from the Lagrangian (1) as the Euler-Lagrange equations. These equations for fields directly generate equations for the Wightman functions. For simplicity we will restrict here our analysis the case of free fields, so we put $g=0$. Thus for the scalar field we have the Klein-Gordon equation $\left(2 \partial_{+} \partial_{-}+m^{2}\right) S_{\phi \phi}\left(x^{+}, x^{-}\right)=0$, which can be integrated over $x^{+}$variable into the integro-(partial) differential (IPD) equation for $S_{\phi \phi}\left(x^{+}, x^{-}\right)$

$$
\partial_{-} S_{\phi \phi}\left(x^{+}, x^{-}\right)=-\frac{1}{4 \pi} \frac{1}{x^{-}-i 0}-\frac{m^{2}}{2} \int_{0}^{x^{+}} d \tau S_{\phi \phi}\left(\tau, x^{-}\right) .
$$

The Lorentz transformation (7a) in the infinitesimal case leads to the condition $\left(x^{+} \partial_{+}-x^{-} \partial_{-}\right) S_{\phi \phi}\left(x^{+}, x^{-}\right)=$ 0 , which can be used with the analyticity condition to transform (14) into another IPD equation for $S_{\phi \phi}\left(x^{+}, x^{-}\right)$

$$
\partial_{+} S_{\phi \phi}\left(x^{+}, x^{-}\right)=-\frac{1}{4 \pi} \frac{1}{x^{+}-i 0}-\frac{m^{2}}{2} \int_{0}^{x^{-}} d \xi S_{\phi \phi}\left(x^{+}, \xi\right) .
$$

These IPD equations can be solved in terms of the Fourier integral as

$$
S_{\phi \phi}\left(x^{+}, x^{-}\right)=\int_{0}^{\infty} \frac{d k^{+}}{4 \pi k^{+}} \exp \left(-i k^{+} x^{-}\right) \exp \left(-i \frac{m^{2}}{2 k^{+}} x^{+}\right),
$$

which is a well-known expression [7], but here we have obtained it without introducing Fourier representation of $\phi$ in terms of creation and annihilation operators. Further we find the Volterra integral equation for $S_{\phi \phi}\left(x^{+}, x^{-}\right)$ [8]

$$
S_{\phi \phi}\left(x^{+}, x^{-}\right)=-\frac{1}{4 \pi} \ln \left(m^{2}\left|x^{+} x^{-}\right|\right)-\frac{i}{8}\left[\operatorname{sgn}\left(x^{+}\right)+\operatorname{sgn}\left(x^{-}\right)\right]-\frac{m^{2}}{2} \int_{0}^{x^{+}} d \tau \int_{0}^{x^{-}} d \xi S_{\phi \phi}(\tau, \xi),
$$

which explicitly shows, that the Wightman function $S_{\phi \phi}\left(x^{+}, x^{-}\right)$is logarithmically divergent at $x^{+} \rightarrow 0$ or $x^{-} \rightarrow 0$. Also we find the mass dependence relation

$$
m^{2} \frac{\partial}{\partial m^{2}} S_{\phi \phi}\left(x^{+}, x^{-}\right)=x^{+} \partial_{+} S_{\phi \phi}\left(x^{+}, x^{-}\right)=-\frac{1}{4 \pi}-\frac{m^{2}}{2} x^{+} \int_{0}^{x^{-}} d \xi S_{\phi \phi}\left(x^{+}, \xi\right)
$$

which indicates that the mass dependent part of $S_{\phi \phi}$ is regular and non-vanishing at $x^{ \pm}=0$. This agrees with the observation [9], that at the LF hypersurface there is no scaling symmetry for massive scalar fields.

Analogous analysis can be carried out for the fermion field, and starting from the Dirac equation (dynamical equation) for a free field case $i \sqrt{2} \partial_{+} S_{++}(x)=M S_{-+}(x)$, one finds the integral equation

$$
i \sqrt{2} S_{++}\left(x^{+}, x^{-}\right)=\frac{1}{2 \pi} \frac{1}{x^{-}-i 0}+M \int_{0}^{x^{+}} d \tau S_{-+}\left(\tau, x^{-}\right)
$$

But another Dirac equation (non-dynamical equation) $i \sqrt{2} \partial_{-} S_{-+}(x)=M S_{++}(x)$ leads to the IPD equation for $S_{-+}(x)$

$$
\partial_{-} S_{-+}\left(x^{+}, x^{-}\right)=-\frac{M}{4 \pi} \frac{1}{x^{-}-i 0}-\frac{M^{2}}{2} \int_{0}^{x^{+}} d \tau S_{-+}\left(\tau, x^{-}\right),
$$

while the Lorentz symmetry condition $\left(x^{+} \partial_{+}-x^{-} \partial_{-}\right) S_{-+}\left(x^{+}, x^{-}\right)=0$ leads to another IPD equation for $S_{-+}(x)$

$$
\partial_{+} S_{-+}\left(x^{+}, x^{-}\right)=-\frac{M}{4 \pi} \frac{1}{x^{+}-i 0}-\frac{M^{2}}{2} \int_{0}^{x^{-}} d \xi S_{-+}\left(x^{+}, \xi\right) .
$$

Similarly we find the integral equation

$$
i \sqrt{2} S_{--}\left(x^{+}, x^{-}\right)=\frac{1}{2 \pi} \frac{1}{x^{+}-i 0}+M \int_{0}^{x^{-}} d \xi S_{-+}\left(x^{+}, \xi\right)
$$


and the equality of the Wightman functions $S_{+-}(x)=S_{-+}(x)$. These equations can be solved for the Wightman functions $S_{a b}$ in terms of the Fourier integral representation

$$
\begin{aligned}
& S_{++}\left(x^{+}, x^{-}\right)=\sqrt{2} \int_{0}^{\infty} \frac{d k^{+}}{4 \pi} \exp \left(-i k^{+} x^{-}\right) \exp \left(-i \frac{M^{2}}{2 k^{+}} x^{+}\right), \\
& S_{-+}\left(x^{+}, x^{-}\right)=\frac{M}{\sqrt{2}} \int_{0}^{\infty} \frac{d k^{+}}{4 \pi k^{+}} \exp \left(-i k^{+} x^{-}\right) \exp \left(-i \frac{M^{2}}{2 k^{+}} x^{+}\right), \\
& S_{--}\left(x^{+}, x^{-}\right)=\sqrt{2}\left(\frac{M}{\sqrt{2}}\right)^{2} \int_{0}^{\infty} \frac{d k^{+}}{4 \pi\left(k^{+}\right)^{2}} \exp \left(-i k^{+} x^{-}\right) \exp \left(-i \frac{M^{2}}{2 k^{+}} x^{+}\right),
\end{aligned}
$$

which agree with [7]. For completeness we find the Volterra equation for $S_{-+}(x)$

$$
S_{-+}\left(x^{+}, x^{-}\right)=-\frac{M}{4 \pi} \ln \left(M^{2}\left|x^{+} x^{-}\right|\right)-i \frac{M}{8}\left[\operatorname{sgn}\left(x^{+}\right)+\operatorname{sgn}\left(x^{-}\right)\right]-\frac{M^{2}}{2} \int_{0}^{x^{+}} d \tau \int_{0}^{x^{-}} d \xi S_{-+}(\tau, \xi) .
$$

These integral equations explain why setting $x^{+}=0$ for $S_{-+}$and $S_{--}$inevitably leads to singular integrals over $k^{+}$. Moreover they allow for a finite limit for the massless fermions $M \rightarrow 0$

$$
i \sqrt{2} S_{++}\left(x^{-}\right)=\frac{1}{2 \pi} \frac{1}{x^{-}-i 0}, \quad i \sqrt{2} S_{--}\left(x^{+}\right)=\frac{1}{2 \pi} \frac{1}{x^{+}-i 0}, \quad S_{-+}(x)=S_{+-}(x)=0
$$

The anti-commutator functions can be expressed as the linear combination of Wightman functions, thus we have $\left\langle 0\left|\left\{\psi_{-}(x), \psi_{-}^{\dagger}(0)\right\}\right| 0\right\rangle=S_{--}(x)+S_{--}(-x)$, which satisfies the integral equation

$$
i \sqrt{2}\left\langle 0\left|\left\{\psi_{-}(x), \psi_{-}^{\dagger}(0)\right\}\right| 0\right\rangle=i \delta\left(x^{+}\right)+\frac{M^{2}}{\sqrt{2}} \int_{0}^{x^{-}} d \xi \Delta\left(x^{+}, \xi ; M\right)
$$

with the Lorentz invariant Jordan-Pauli function

$$
\Delta\left(x^{+}, x^{-} ; M\right)=-i \int_{0}^{\infty} \frac{d k^{+}}{2 \pi k^{+}} \sin \left(k^{+} x^{-}+\frac{M^{2}}{2 k^{+}} x^{+}\right) .
$$

This shows that the anti-commutator $\left\{\psi_{-}(x), \psi_{-}^{\dagger}(0)\right\}$ is divergent at $x^{+}=0$ and the corresponding Dirac anti-bracket does not exists.

\section{Gaussian Effective Potential}

Usually for the LF variational calculations one introduces momentum cut-off in the Fourier representation of fields $[10,11]$. However we notice that the vacuum expectation value of the LF Hamiltonian density operator (2), due to the translation symmetry, is

$$
\begin{aligned}
\left\langle 0\left|\mathscr{H}_{L F}(x)\right| 0\right\rangle= & -i \sqrt{2}\left\langle 0\left|\psi_{-}^{\dagger}(0) \partial_{-} \psi_{-}(0)\right| 0\right\rangle+\frac{m^{2}}{2}\langle 0|\phi(0) \phi(0)| 0\rangle+M\left\langle 0\left|\psi_{+}^{\dagger}(0) \psi_{-}(0)\right| 0\right\rangle+ \\
& +M\left\langle 0\left|\psi_{-}^{\dagger}(x) \psi_{+}(0)\right| 0\right\rangle+g\left[\left\langle 0\left|\psi_{+}^{\dagger}(0) \phi(0) \psi_{-}(0)\right| 0\right\rangle+\left\langle 0\left|\psi_{-}^{\dagger}(0) \phi(0) \psi_{+}(0)\right| 0\right\rangle\right] .
\end{aligned}
$$

Since this is a linear combination of the Wightman functions for fields at the coinciding points, thus it is a singular expression. For a regularization we take the point-splitting procedure e.g. $\left\langle 0\left|\psi_{+}^{\dagger}(0) \psi_{-}(0)\right| 0\right\rangle \rightarrow$ $\left\langle 0\left|\psi_{+}^{\dagger}(0) \psi_{-}(x)\right| 0\right\rangle$. The Hartree approximation, which leads to the Gaussian effective potential, uses free quantum field operators with masses, which are the variational parameters: $\mu_{b}$ for bosons, $\mu_{f}$ for fermions. Also the scalar field may have a non-vanishing vacuum expectation value $\langle 0|\phi(x)| 0\rangle=\phi_{0}$ and the 3-point Wightman 
functions factorize $\left\langle 0\left|\psi_{ \pm}^{\dagger}(0) \phi(0) \psi_{\mp}(x)\right| 0\right\rangle=\phi_{0}\left\langle 0\left|\psi_{ \pm}^{\dagger}(0) \psi_{\mp}(x)\right| 0\right\rangle$. Thus the Hartree approximation with the point splitting regularization gives

$$
\begin{aligned}
\left\langle 0\left|\mathscr{H}_{L F}\right| 0\right\rangle_{\text {reg }}^{\mathrm{Har}} & =-i \sqrt{2} \partial_{-}^{x} S_{--}\left(-x ; \mu_{f}\right)+\frac{m^{2}}{2} S_{\phi \phi}\left(-x ; \mu_{b}\right)+\left(M+g \phi_{0}\right)\left[S_{+-}\left(-x ; \mu_{f}\right)+S_{-+}\left(-x ; \mu_{f}\right)\right] \\
& =\left[2\left(M+g \phi_{0}\right)-\mu_{f}\right] S_{+-}\left(-x ; \mu_{f}\right)+\frac{m^{2}}{2} S_{\phi \phi}\left(-x ; \mu_{b}\right)
\end{aligned}
$$

and similarly we define $\left\langle 0\left|T^{++}\right| 0\right\rangle_{\text {reg }}^{\mathrm{Har}}=i \sqrt{2} \partial_{-}^{x} S_{++}\left(-x, \mu_{f}\right)-\partial_{-}^{2} S_{\phi \phi}\left(-x ; \mu_{b}\right)$. The Hartree effective potential is the linear combination of these expressions with an arbitrary parameter $\alpha$ as $V_{e f f}\left[\mu_{b}, \mu_{f}, x^{+}, x^{-}\right]=$ $\left\langle 0\left|\left(\mathscr{H}_{L F}+\alpha T^{++}\right)\right| 0\right\rangle_{\text {reg }}^{\mathrm{Har}}$. The optimum values of $\mu_{b}, \mu_{f}$, which are regularization independent $\mu_{b}=m$ and $\mu_{f}=M+g \phi_{0}$, imply two conditions $\alpha x^{+}+x^{-}=0$ and $\operatorname{sgn}\left(x^{+}\right)+\operatorname{sgn}\left(x^{-}\right)=0$. Thus $\alpha>0$ and the interval $x^{2}=2 x^{+} x^{-}=-\alpha\left(x^{+}\right)^{2}<0$. Accordingly only a space-like point-splitting regularization is consistent.

\section{Conclusions and Prospects}

There is no classical theory underlying the Lorentz covariant LF Yukawa model in $D=1+1$. The LF Wightman functions for free scalar and fermion fields can be obtained without introducing the Fourier representation for fields. Generalization to the LF Yukawa model in $D=3+1$ seems to be straightforward. The LF variational calculations for the Lorentz covariant models are possible with a space-like point-splitting regularization. It is interesting to check how the novel LF formulation works in the exactly solvable models.

Acknowledgments We would like to thank the organizers of the LC2014 Workshop for all their efforts and hospitality.

Open Access This article is distributed under the terms of the Creative Commons Attribution License which permits any use, distribution, and reproduction in any medium, provided the original author(s) and the source are credited.

\section{Appendix A: Definitions and Basic Assumptions About the Light Front}

The LF coordinates are defined as $x^{ \pm}=\left(x^{0} \pm x^{3}\right) / \sqrt{2}$ and the partial derivatives are denoted as $\partial_{ \pm}=\partial / \partial x^{ \pm}$. The metric components are $g_{+-}=g_{-+}=1, \quad g_{++}=g_{--}=0$. The Dirac matrices $\gamma^{ \pm}$are taken in the representation, which leads to the simplest projection operators $\Lambda_{ \pm}$

$$
\gamma^{+}=\sqrt{2}\left[\begin{array}{ll}
0 & 1 \\
0 & 0
\end{array}\right], \quad \gamma^{-}=\sqrt{2}\left[\begin{array}{ll}
0 & 0 \\
1 & 0
\end{array}\right], \quad \Lambda_{+}=\frac{\gamma^{-} \gamma^{+}}{2}=\left[\begin{array}{ll}
0 & 0 \\
0 & 1
\end{array}\right], \quad \Lambda_{-}=\frac{\gamma^{+} \gamma^{-}}{2}=\left[\begin{array}{ll}
1 & 0 \\
0 & 0
\end{array}\right] .
$$

The fermion fields $\Psi$ is projected into two components $\psi_{ \pm}$as

$$
\Lambda_{+} \Psi=\left[\begin{array}{c}
0 \\
\psi_{+}
\end{array}\right], \quad \Lambda_{-} \Psi=\left[\begin{array}{c}
\psi_{-} \\
0
\end{array}\right], \quad \Psi^{\dagger}=\left[\begin{array}{ll}
\psi_{-}^{\dagger} & \psi_{+}^{\dagger}
\end{array}\right]
$$

The Lorentz boost transformation $x^{\prime \mu}=\Lambda_{\nu}^{\mu} x^{\nu}$ is generated by the parameter $\omega_{-+}=\omega$ with $\Lambda_{+}^{+}=e^{\omega}, \Lambda_{-}^{-}=$ $e^{-\omega}, \Lambda_{-}^{+}=\Lambda_{+}^{-}=0$. The Lorentz invariance of the Minkowski metric follows immediately $g_{\mu \nu} \Lambda_{\alpha}^{\mu} \Lambda_{\beta}^{v}=$ $g_{\alpha \beta}$ and the coordinates transform uniformly $x^{\prime+}=e^{\omega} x^{+}, x^{\prime-}=e^{-\omega} x^{-} \cdot x^{\prime \pm}=e^{ \pm \omega} x^{ \pm}$The (spin) matrix $S(\Lambda)$ is defined by the relation $S(\Lambda) \gamma^{\mu} S^{-1}(\Lambda)=\gamma^{\nu} \Lambda_{v}^{\mu}$, which in $D=1+1$ dimensions looks as

$$
S^{-1}(\omega) \gamma^{+} S(\omega)=\Lambda_{+}^{+} \gamma^{+}=e^{\omega} \gamma^{+}, \quad S^{-1}(\omega) \gamma^{-} S(\omega)=\Lambda_{-}^{-} \gamma^{-}=e^{-\omega} \gamma^{-},
$$

and leads to

$$
S(\omega)=e^{\omega / 2} \Lambda_{+}+e^{-\omega / 2} \Lambda_{-}=\left[\begin{array}{cc}
e^{-\omega / 2} & 0 \\
0 & e^{\omega / 2}
\end{array}\right]
$$


The Lorentz transformation for the fermion $\Psi(x), \bar{\Psi}(x)$ and scalar $\phi(x)$ quantum fields is generated by the unitary operator $U(\omega)$ :

$$
\begin{aligned}
& U(\omega) \Psi(x) U^{-1}(\omega)=S^{-1}(\omega) \Psi\left(x^{\prime}\right), \quad U(\omega) \bar{\Psi}(x) U^{-1}(\omega)=\bar{\Psi}\left(x^{\prime}\right) S(\omega), \\
& U(\omega) \phi(x) U^{-1}(\omega)=\phi\left(x^{\prime}\right),
\end{aligned}
$$

thus explicitly we have

$$
\begin{array}{rlrl}
U(\omega) \psi_{+}(x) U^{-1}(\omega) & =e^{-\omega / 2} \psi_{+}\left(e^{\omega} x^{+}, e^{-\omega} x^{-}\right), & & U(\omega) \psi_{-}(x) U^{-1}(\omega)=e^{\omega / 2} \psi_{-}\left(e^{\omega} x^{+}, e^{-\omega} x^{-}\right), \\
U(\omega) \psi_{+}^{\dagger}(x) U^{-1}(\omega) & =e^{\omega / 2} \psi_{+}^{\dagger}\left(e^{\omega} x^{+}, e^{-\omega} x^{-}\right), & & U(\omega) \psi_{-}^{\dagger}(x) U^{-1}(\omega)=e^{-\omega / 2} \psi_{-}^{\dagger}\left(e^{\omega} x^{+}, e^{-\omega} x^{-}\right), \\
U(\omega) \phi(x) U^{-1}(\omega) & =\phi\left(e^{\omega} x^{+}, e^{-\omega} x^{-}\right) .
\end{array}
$$

\section{Appendix B: Light-Front Dirac-Bergmann Procedure}

The Lorentz invariant Lagrangian density for the Yukawa model given by (1) leads to the canonical momenta

$$
\pi_{+}^{\dagger}=\frac{\partial \mathscr{L}}{\partial \partial_{+} \psi_{+}}=-i \sqrt{2} \psi_{+}^{\dagger}, \quad \pi_{-}^{\dagger}=\frac{\partial \mathscr{L}}{\partial \partial_{+} \psi_{-}}=0, \quad \pi_{-}=\frac{\partial \mathscr{L}}{\partial \partial_{+} \psi_{-}^{\dagger}}=0, \quad \pi_{\phi}=\frac{\partial \mathscr{L}}{\partial \partial_{+} \phi}=\partial_{-} \phi,
$$

where for the anti-commuting variables the left-hand differentiation convention is applied. There are three primary constraints $\Phi_{1}=\pi_{-}^{\dagger} \approx 0, \Phi_{2}=\pi_{-} \approx 0, \Phi_{3}=\pi_{\phi}-\partial_{-} \phi \approx 0$. The canonical Hamiltonian density, which follows from the generalized Legendre transformation

$$
\mathscr{H}_{c a n}=\pi_{\phi} \partial_{+} \phi-\pi_{+}^{\dagger}\left(\partial_{+} \psi_{+}\right)-\mathscr{L}=\frac{m^{2}}{2} \phi^{2}-i \sqrt{2} \psi_{-}^{\dagger} \partial_{-} \psi_{-}+(M+g \phi)\left(\psi_{+}^{\dagger} \psi_{-}+\psi_{-}^{\dagger} \psi_{+}\right),
$$

leads by the stationarity conditions to the secondary constraints $\Phi_{4}=i \sqrt{2} \partial_{-} \psi_{-}^{\dagger}+(M+g \phi) \psi_{+}^{\dagger} \approx 0, \quad \Phi_{5}=$ $i \sqrt{2} \partial_{-} \psi_{-}-(M+g \phi) \psi_{+} \approx 0$. These constraints are usually interpreted as non-dynamical Dirac equations, which allow to express the non-dynamical components $\psi_{-}, \psi_{-}^{\dagger}$ in terms of the dynamical fields. One calculates the matrix of Poisson brackets for constraints $\mathscr{M}_{a b}\left(x^{-}, y^{-}\right)=\left\{\Phi_{a}\left(x^{+}, y^{-}\right), \Phi_{b}\left(x^{+}, x^{-}\right\}_{P}\right.$

$$
\mathscr{M}_{a b}\left(x^{-}, y^{-}\right)=\left[\begin{array}{ccccc}
0 & 0 & 0 & 0 & i \sqrt{2} \partial_{-}^{x} \\
0 & 0 & 0 & i \sqrt{2} \partial_{-}^{x} & 0 \\
0 & 0 & -2 \partial_{-}^{x} & -g \psi_{+}^{\dagger}\left(y^{-}\right) & g \psi_{+}\left(y^{-}\right) \\
0 & -i \sqrt{2} \partial_{-}^{x} & g \psi_{+}^{\dagger}\left(y^{-}\right) & 0 & \mathscr{A}\left(x^{-}, y^{-}\right) \\
-i \sqrt{2} \partial_{-}^{x} & 0 & -g \psi_{+}\left(y^{-}\right) & -\mathscr{A}\left(x^{-}, y^{-}\right) & 0
\end{array}\right] \delta\left(x^{-}-y^{-}\right),
$$

where $\mathscr{A}\left(x^{-}, y^{-}\right)=i / \sqrt{2}\left[M+g \phi\left(x^{-}\right)\right]\left[M+g \phi\left(y^{-}\right)\right]$. This matrix contains fields variables thus finding its inverse is highly a non-trivial task. Therefore we consider here only a free field case, $g=0$ which leads to the inverse matrix

$$
\mathscr{M}_{a b}^{-1}\left(x^{-}, y^{-}\right)=\left[\begin{array}{ccccc}
0 & \mathfrak{c}\left(x^{-}-y^{-}\right) & 0 & 0 & -\mathfrak{a}\left(x^{-}-y^{-}\right) \\
-\mathfrak{c}\left(x^{-}-y^{-}\right) & 0 & 0 & -\mathfrak{a}\left(x^{-}-y^{-}\right) & 0 \\
0 & 0 & \mathfrak{b}\left(x^{-}-y^{-}\right) & 0 & 0 \\
0 & \mathfrak{a}\left(x^{-}-y^{-}\right) & 0 & 0 & 0 \\
\mathfrak{a}\left(x^{-}-y^{-}\right) & 0 & 0 & 0 & 0
\end{array}\right],
$$

where functions $\mathfrak{a}, \mathfrak{c}, \mathfrak{b}$ are not determined uniquely, but they have to satisfy the differential equations

$$
i \sqrt{2} \partial_{-} \mathfrak{a}\left(x^{-}\right)=\delta\left(x^{-}\right), \quad 2 \partial_{-} \mathfrak{c}\left(x^{-}\right)=-M^{2} \mathfrak{a}\left(x^{-}\right), \quad 2 \partial_{-} \mathfrak{b}\left(x^{-}\right)=-\delta\left(x^{-}\right) .
$$


Accordingly here the Dirac procedure is not a complete algorithm for finding Dirac brackets thus one needs to add some additional assumptions for them. We may evaluate two Dirac brackets

$$
\begin{aligned}
& \left\{\psi_{+}^{\dagger}\left(x^{+}, x^{-}\right), \psi_{+}\left(x^{+}, y^{-}\right)\right\}_{D}=-\frac{i}{\sqrt{2}} \delta\left(x^{-}-y^{-}\right), \\
& \left\{\phi\left(x^{+}, x^{-}\right), \phi\left(x^{+}, y^{-}\right)\right\}_{D}=\mathfrak{b}\left(x^{-}-y^{-}\right)=-\frac{1}{4} \operatorname{sgn}\left(x^{-}-y^{-}\right),
\end{aligned}
$$

where from the the antisymmetry of Dirac bracket we can impose the condition $\mathfrak{b}\left(x^{-}-y^{-}\right)=-\mathfrak{b}\left(y^{-}-x^{-}\right)$. Other Dirac brackets remain undefined

$$
\begin{aligned}
& \left\{\psi_{-}\left(x^{+}, x^{-}\right), \psi_{+}^{\dagger}\left(x^{+}, y^{-}\right)\right\}_{D}=\left\{\psi_{+}\left(x^{+}, x^{-}\right), \psi_{-}^{\dagger}\left(x^{+}, y^{-}\right)\right\}_{D}=-i \frac{M}{\sqrt{2}} \mathfrak{a}\left(x^{-}-y^{-}\right), \\
& \left\{\psi_{-}\left(x^{+}, x^{-}\right), \psi_{-}^{\dagger}\left(x^{+}, y^{-}\right)\right\}_{D}=-\mathfrak{c}\left(x^{-}-y^{-}\right),
\end{aligned}
$$

Usually one argues that some boundary conditions are needed for fixing these Dirac brackets. However such boundary conditions are not physically self-evident and they may spoil the Lorentz symmetry of the model. Moreover any additional condition, which allows to fix $\mathfrak{a}$ and $\mathfrak{c}$ uniquely, leads to well-defined Dirac brackets for all fields taken at equal LF time $x^{+}$.

\section{References}

1. Burkardt, M.: Light front quantization. Adv. Nucl. Phys. 23, 1-74 (1996)

2. Brodsky, S.J., Pauli, H.-C., Pinsky, S.: Quantum chromodynamics and other field theories on the light cone. Phys. Lett. C (Phys. Rep.) 301, 299-486 (1998)

3. Heinzl, T.: Lecture Notes in Physics, vol. 572. pp. 55 Springer, Berlin (2001)

4. Dirac, P.A.M.: Generalized Hamiltonian dynamics. Can. J. Phys. 2, 129-148 (1950)

5. Dirac, P.A.M.: Lectures on Quantum Mechanics. Yeshiva University Press, New York (1964)

6. Steinhardt, P.J.: Problems of quantization in the infinite momentum frame. Ann. Phys. 128, $425-447$ (1980)

7. Martinovic, L., Luban, M.: Analytic solution of the microcausality problem in discretized light cone quantization. Phys. Lett. B 605, 203-213 (2005)

8. Przeszowski, J.A.: Lorentz symmetry for the light-front Wightman functions. Acta Phys. Pol. Proc. Suppl. B 6, 327333 (2013)

9. Przeszowski, J.A., Żochowski, J.: Scale and lorentz transformations at the light-front. Few Body Syst. 55, 485-491 (2014)

10. Harindranath, A., Vary, J.P.: Variational calculation of the spectrum of two-dimensional $\phi^{4}$ theory in light-front field theory. Phys. Rev. D 37, 3010-3013 (1988)

11. Bartnik, E.A., Głazek, St.: Light-front variational approach to scalar field theories. Phys. Rev. D 39, 1249-1250 (1989) 